\section{El análisis estratigráfico constructivo como documentación de la materialidad y guía para su conservación en el proyecto de restauración: las Torres de Serranos de Valencia y la Torre del Homenaje del Castillo de Cofrentes (Valencia)}

Camilla Mileto, Fernando Vegas

Universidad Politécnica de Valencia

\begin{abstract}
Resumen
En el texto se presentan dos casos donde la aplicación del análisis estratigráfico constructivo representó, además de la posibilidad de realizar una hipótesis de fases constructivas, la herramienta utilizada para el estudio de la materialidad del monumento (materiales, técnicas constructivas, etc.) y la guía para la realización de un proyecto de restauración y una obra más consciente de la materialidad misma. En el primer caso, el estudio de las Torres de Serranos, enteramente construidas en fábrica de sillería, la interpretación de los datos materiales implicó la combinación del método del análisis estratigráfico con criterios cronotipológicos. En el segundo caso, en la torre del Homenaje del Castillo de Cofrentes, además del análisis estratigráfico constructivo se realizó el proyecto de restauración. En esa ocasión se tuvo por tanto la posibilidad de reflexionar sobre la conservación de los datos estratigráficos como elementos fundamentales de la conservación de la materialidad del edificio.
\end{abstract}

Palabras clave: estratigrafía, proyecto de restauración, arquitectura, sillería, cronotipología

\begin{abstract}
This text presents two cases where the application of stratigraphic wall analysis represented, in addition to the possibility of constructing a hypothesis of construction phases, the tool used for the study of the materiality of the monument (materials, construction techniques, etc.) and a guideline for carrying out a more materiality-conscious restoration project and work. In the first case, the study of the Torres de Serranos, which are constructed entirely of stonework, the interpretation of material data meant a combination of the stratigraphic analysis method with chronotypological criteria. In the second case, a tower of the Homenaje del Castillo de Confrentes, in addition to stratigraphic wall analysis the restoration project was also carried out. On this particular occasion it was possible to reflect on the conservation of stratigraphic data as fundamental elements in the conservation of the building's materiality.
\end{abstract}

Key words: stratigraphy, restoration project, architecture, stonemasonry, chronotypology

\section{EL ANÁLISIS ESTRATIGRÁFICO CONSTRUCTIVO DE UN EDIFICIO MONUMENTAL EN FÁBRICA DE SILLERÍA:LAS TORRES DE SERRANOS DE VALENCIA ${ }^{1}$}

El análisis estratigráfico de las Torres de Serranos se ha realizado, por encargo del Ayuntamiento de Valencia, dentro del marco del Estudio Previo de Diagnosis al Proyecto de Limpieza y Consolidación del edificio. De esta guisa, el estudio estratigráfico ha podido apoyarse en una serie de otros estudios, tanto para la recogida de los datos como para la posterior interpretación. Los análisis realizados para la determinación de los tipos de piedra ${ }^{2}$ y morteros ${ }^{3}$ presentes en el edificio, los estudios históricos detallados ${ }^{4}$ y la documentación precisa de la posición y tipos de marcas de cantería ${ }^{5}$, han facilitado la tarea del estudio estratigráfico.

\subsection{Las Torres de Serranos de Valencia}

Se trata de un grandioso edificio que asume al mismo tiempo un carácter defensivo y un carácter representativo. Se trata de hecho del acceso a la ciudad desde norte. No obstante su aspecto de construcción sólida y masiva, la monumental puerta se construyó en un tiempo relativamente breve, entre 1392 y 1398, de mano del maestro Pere Balaguer. El edificio consta de dos grandes torres almenadas, un tiempo conectadas a las murallas, y de un cuerpo central más bajo, que permite, a través de un gran arco de medio punto, la entrada a la ciudad. En el interior, en cada torre se abren tres naves en los tres diferentes niveles, mientras que el cuerpo central constituye el espacio de conexión. Desde la planta baja al primer piso se accede por una majestuosa escalera situada en el lado sur, mientras que al segundo piso se accede por escaleras menores internas a las naves.

En 1586, a causa del incendio de las cárceles de la ciudad y la necesidad de trasladar los presos a diferente sitio, las

\footnotetext{
1 El análisis estratigráfico constructivo de las Torres de Serranos ha sido realizado por los autores por encargo del arquitecto Francisco Cervera Arias responsable del equipo técnico y encargado del Estudio de Diagnosis y del Proyecto de Limpieza y Consolidación de las Torres de Serranos. El encargo ha sido adjudicado mediante concurso público, por el Ayuntamiento de Valencia a la empresa constructora EXISA, en el año 1999. El análisis estratigráfico constructivo ha sido realizado durante el año 2000.

2 Los análisis de los tipos de piedra han sido realizados por Jorge Obrado, geólogo, en la Universidad de Zaragoza y por el laboratorio AIDICO de Valencia.

3 Los análisis de componentes y granulometría de los morteros han sido realizados por el laboratorio SEG de Valencia.

4 Los estudios históricos han sido desarrollados por: Amadeo Serra Desfilis, José Luis Cervera Torrejón, Carmen Blázquez Izquierdo, Daniel Benito Goerlich y Ignasi Corresa Martín.

5 El estudio y levantamiento de los tipos y ubicación de las marcas de cantería ha sido realizado por: José Manuel Martínez García, arqueólogo, y Miguel Micó Chofré.
} 
Torres se transformaron en prisión. La adecuación del edificio a cárcel supuso la construcción de una serie de forjados interiores, para multiplicar el espacio donde encerrar a los presos, y de unas paredes de cierre de las naves interiores, antes abiertas hacia la ciudad. En 1868, se destruyó el conjunto de las murallas de Valencia, incluidas las puertas fortificadas, con exclusión de las Torres de Serranos y las Torres de Quart, entonces todavía utilizadas como prisión.

Fue en 1887 cuando se trasladó nuevamente la prisión de la ciudad, liberando las Torres de Serranos. De este momento en adelante, el edificio fue sometido a una serie de intervenciones dirigidas a la recuperación del antiguo esplendor de las Torres, después de trescientos años de cárcel. En el mismo año de la liberación, se demuelen, por tanto, las paredes que clausuraban las naves y los forjados interiores.

En 1893, gracias a un informe favorable de la Real Academia de San Carlos, se reabrió el foso, se quitaron las rejas metálicas, y se reconstruyeron el adarve y la tan discutida escalera principal de acceso, objeto de debate entre la Academia y el Ayuntamiento, por causa de su falta de clara contemporaneidad con el resto del edificio. En este periodo, fue de gran peso la presencia de José Aixa, escultor, encargado de la restauración de edificio entre 1893 y 1914.

En 1931, el edificio se declara monumento nacional y durante la guerra civil sirve como almacén de obras de arte hasta que, en 1974, se destina a museo marítimo de la ciudad, sufriendo de ese momento en adelante una serie de pequeñas intervenciones de reparación.

Se trata evidentemente de un edificio que, en su conjunto, se ha mantenido intacto en el tiempo. Sin embargo, sobre todo el periodo en el que se destinó a cárcel, marcó profundamente el edificio, imponiendo, a finales del siglo XIX, una serie de restauraciones, o reconstrucciones de notable envergadura que todavía caracterizan su aspecto.

\subsection{La metodología adoptada en el análisis estratigráfico constructivo (Fig. 1 y 2)}

Aunque en el análisis estratigráfico constructivo de las Torres de Serranos se siguió, por lo menos a grandes rasgos, la metodología general de este tipo de estudio. Sin embargo, las
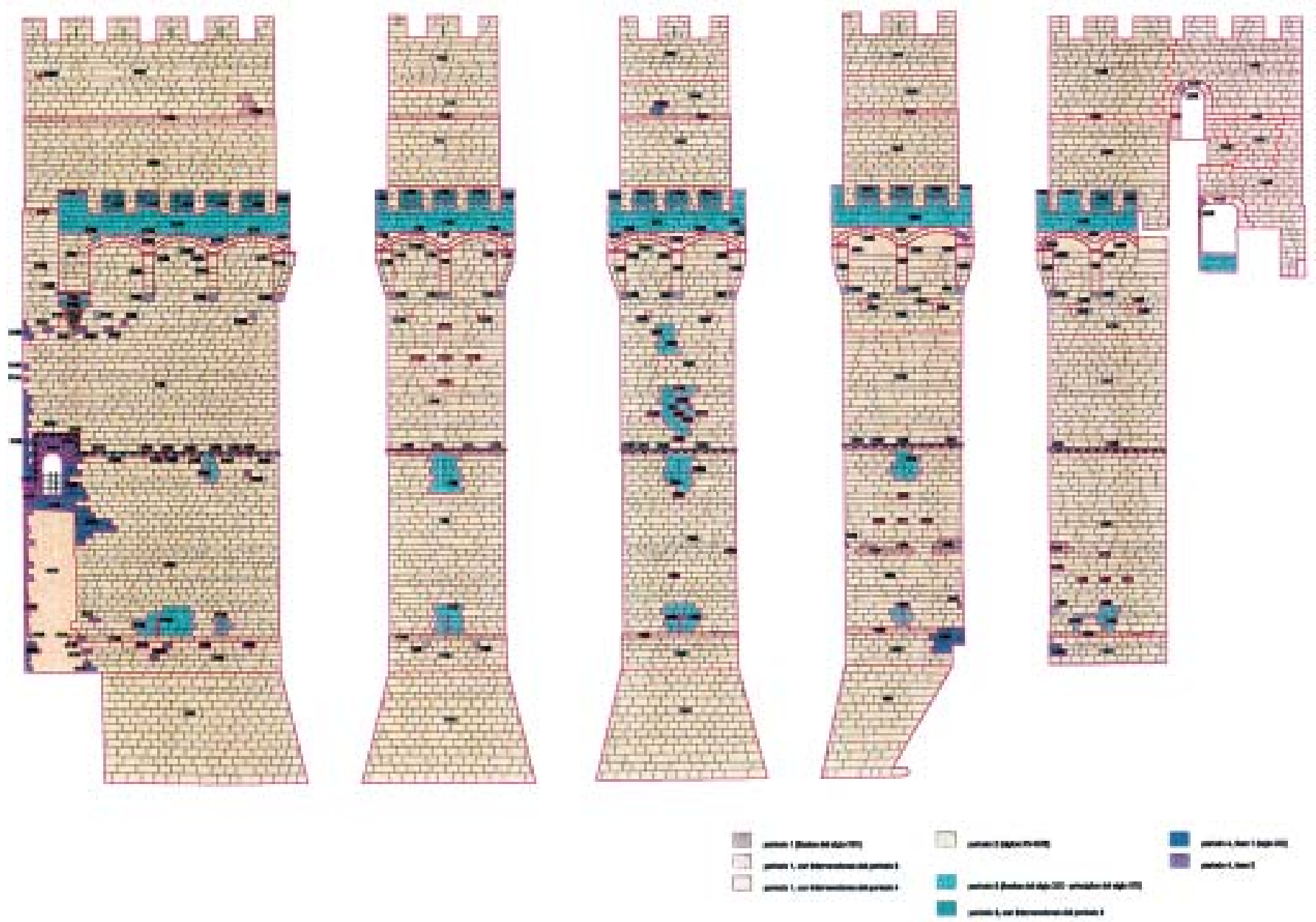

Fig. 1. Plano de hipótesis de períodos constructivos de la torre de Poniente de las Torres de Serranos de Valencia 


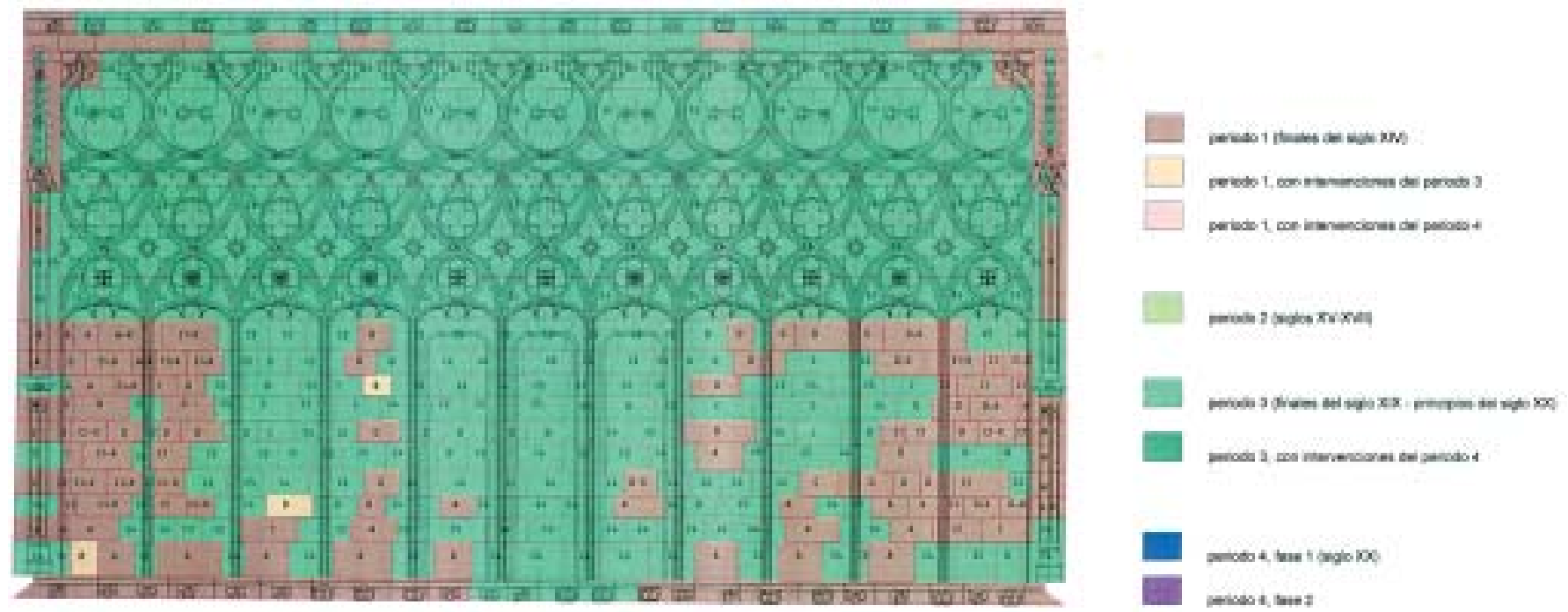

Fig. 2. Plano de hipótesis de períodos constructivos de la tracería del cuerpo central de las Torres de Serranos de Valencia

particularidades del propio edificio impusieron una serie de modificaciones o adaptaciones. Las Torres de Serranos, de hecho, están completamente construidas con fábricas de sillería, o mejor dicho, se trata de muros de dos paramentos de sillares recibidos con mortero y con un relleno intermedio, posiblemente de un hormigón de cal. Por tanto, al no existir enlucidos, cuyas superposiciones indicaran las diferentes intervenciones, el estudio requirió una adaptación del método a la especificidad del caso.

Una fábrica de sillería, por su misma naturaleza, constituye el conjunto de un gran número de sillares que no observan ningún tipo de relación física directa entre ellos. La relación entre dos sillares se estableció a través del mortero que los une de manera que, si el mortero fuera contemporáneo a los sillares, se podría considerar el conjunto como una única unidad estratigráfica. Por el contrario, en el caso en el cual el mortero fuera posterior a los dos sillares, no se podría afirmar la contemporaneidad de los mismos y se tendrían que tratar como dos diferentes unidades estratigráficas. En este segundo caso, para demostrar esa relación de contemporaneidad sería necesario recurrir a un proceso de comparación tipológica basado en la observación de las características de ambos sillares.

En el caso específico de las Torres de Serranos, se intentó una caracterización de los sillares a través la observación del litotipo (se emplearon 5 tipos fundamentales de piedra), de la presencia de pátinas y del nivel de degradación (que originaron varios subtipos además de los cinco tipos de piedra) y de la talla o labra superficial (se identificaron cinco tipos predominantes de labra), además de la presencia o ausencia de marcas de cantería. Todos estos datos se contrastaron con la analítica realizada durante el Es- tudio Previo de Diagnosis. Todos los datos recogidos, con sus variables, se cruzaron entre ellos generando varios tipos de sillares, de manera que se pudieron establecer, en un primer momento, relaciones de contemporaneidad entre los sillares. Las relaciones de anterioridad/posterioridad se establecieron en un segundo momento: por una parte, a través de la observación de las relaciones estratigráficas (cubre/cubierto por, corta/cortado por, se apoya/se le apoya, rellena/rellenado por); por otra parte, mediante relaciones cronotipológicas.

Para los morteros se establecieron varios tipos (seis tipos fundamentales con 11 variaciones), también deducidos de la analítica realizada durante el Estudio Previo de Diagnosis, que se ordenaron entre ellos según relaciones estratigráficas de contemporaneidad, anterioridad/posterioridad.

\section{La ficha de análisis estratigráfico}

Dada la especificidad del caso se organizó una ficha de análisis estratigráfico que recogió la siguiente información: número de identificación de la unidad estratigráfica muraria, descripción de la misma (elemento arquitectónico, posible función), caracterización de la unidad (tipo de piedra, tipo de talla, tipo de mortero, presencia de marcas de cantería), relaciones de contemporaneidad (se une, igual a), relaciones de anterioridad (cortado por, se le apoya, cubierto por, relleno por) y de posterioridad (corta, se apoya, cubre, rellena).

Respecto a la ficha de análisis estratigráfico constructivo que comúnmente utilizamos, se consideró interesante, en este caso, desglosar la descripción de la unidad en dos apartados: descripción de la unidad y caracterización de la misma. De esta forma, en el segundo apartado aparecieron 
de manera más clara los indicadores establecidos para el tipo de material, la talla y las marcas de cantería.

\subsection{Descripción de la hipótesis de los periodos constructivos}

En el presente estudio, se identificaron sustancialmente cuatro periodos de construcción e intervención en el edificio: un primer periodo que corresponde a la fase de construcción de las Torres (1392-1398) y a todo el tiempo que transcurrió hasta su conversión en cárcel en 1586; el segundo periodo corresponde al tiempo en que el edificio fue utilizado como prisión (desde 1586 hasta 1887); el tercer periodo corresponde a varias fases de la intervención de restauración que se realizaron entre finales del siglo XIX y principios del siglo XX (1888-1920); el cuarto periodo corresponde a las intervenciones del siglo $\mathrm{XX}$, sobre todo, desarrolladas entre 1971 y 1990.

Se trata, por tanto, de una primera división en cuatro grandes periodos de manera que cada uno de ellos contiene, a su vez, varias fases de intervención que no siempre se pueden distinguir entre ellas. Además, se debe de precisar que, en este caso, cada intervención no anula las unidades estratigráficas de las fases precedentes sino más bien se superpone con sus unidades de intervención. De manera que bien pocas resultan ser las unidades que pertenecen a un solo periodo, mientras que en la mayoría de los casos se encuentran unidades de un periodo precedente con intervenciones de un periodo posterior.

\section{Periodo 1}

Como se ha dicho anteriormente, el primer periodo corresponde a la fase de construcción del edificio (1392-1398) y a todo el periodo que transcurrió entre el momento en que se terminó la obra y el momento en que el edificio se transformó en cárcel (1586). Las unidades estratigráficas que pertenecen a este periodo se dividieron en tres categorías: en primer lugar, unidades que perteneciendo al primer periodo, no han sufrido ningún tipo de intervención en periodos posteriores y que, por tanto, se conservan en su materialidad primigenia; $y$, en segundo lugar, las unidades que, perteneciendo al primer periodo, han sufrido intervenciones en el tercer periodo (segunda categoría) o en el cuarto periodo (tercera categoría). No se consideraron las intervenciones del segundo periodo dado que no se encontraron aportaciones materiales de las obras realizadas para transformar el edificio en cárcel. No obstante, se pueden leer las huellas de estas intervenciones del segundo periodo en los trabajos de restauración realizados a finales del siglo XIX. A este periodo, por tanto, corresponden la mayoría de las fábricas de las Torres, desde la escarpa, hasta la terraza supe- rior. Perteneciente a este mismo periodo se pueden considerar también las estructuras inferiores del adarve, canes y bóvedas. En muchos sillares se pueden todavía apreciar las marcas de cantería, tanto en el exterior del edificio, como en el interior. Sin embargo, en estas mismas fábricas se realizaron intervenciones sucesivas, sobre todo, de rejuntado y de sustitución o reposición de algunas partes.

\section{Periodo 2}

El segundo periodo ocupa tres siglos, desde que el edificio se transformó en cárcel (1586) hasta que esta se cerró (1887). A este periodo no corresponde en realidad ningún tipo de fábrica dado que la intervención de finales del siglo XIX se planteó la eliminación completa de cualquier añadido de este periodo y la recuperación del estado primigenio de las Torres. Sin embargo, se pueden todavía leer algunas huellas en los paramentos de las naves interiores, posiblemente debidas a las estructuras de los forjados.

\section{Periodo 3}

La mayor parte de las intervenciones se han realizado en el periodo entre finales del siglo XIX y principios del siglo XX. Se trata de obras emprendidas después de la clausura de la cárcel en 1887, con el objetivo de repristinar el estado primigenio de las Torres. Constituyen fundamentalmente operaciones de tapiado de huecos abiertos en el periodo anterior, de reconstrucción de escaleras, de sustitución de sillares degradados y de rejuntado de todas las fábricas, y de la reconstrucción del adarve con sus merlones. De manera especial, cobran importancia las obras de refacción de los elementos decorativos, como los capiteles, canes, cornisas, puertas y ventanas y, en particular, de la tracería de la portada principal.

\section{Periodo 4}

En este periodo, que abarca un tiempo entre 1970 y 1990 , se realizaron numerosas intervenciones de consolidación y mantenimiento, principalmente consistentes en el rejuntado de las fábricas de sillería, y el desmontaje y recolocación de los merlones del adarve que presentaban un estado de peligro de caída. Además se sustituyeron algunas piezas decorativas, como es el caso de la tracería superior de las Torres y de las gárgolas.

\subsection{El análisis estratigráfico constructivo como documentación de la materialidad para el proyecto de restauración}

El estudio de las fábricas realizado mediante el método del análisis estratigráfico constructivo ha permitido proporcionar un conjunto de datos sobre el monumento ligados a la historia de su construcción y a las modificaciones que ha 
sufrido en el transcurso del tiempo. En el ámbito de un estudio previo más amplio el análisis estratigráfico constructivo desempeñó un papel triple: en primer lugar, constituyó un instrumento de lectura de las fases constructivas del monumento cuyos resultados se pudieron enlazar con el estudio histórico documental; en segundo lugar, se utilizó como herramienta de observación minuciosa de la materialidad y enlace con los otros tipos de estudios previos (análisis de materiales, estudios de las técnicas constructivas, estudio de policromías y pátinas, etc.); por último se impuso como guía para la restauración y la conservación de los datos materiales en el proyecto y en la obra de restauración. En relación con esta última reflexión, se debe señalar cómo el análisis estratigráfico constructivo ha permitido descubrir detalles y puntos de especial interés, que han podido ser documentados y conservados durante la obra.

\section{EL ANÁLISIS ESTRATIGRÁFICO CONSTRUCTIVO Y EL PROYECTO DE RESTAURACIÓN:LA TORRE DEL HOMENAJE DEL CASTILLO DE COFRENTES (VALENCIA) ${ }^{6}$}

Desde hace algunos años, se está estudiando y restaurando el Castillo de Cofrentes, donde se han concluido ya una primera y una segunda fase de la intervención de restauración, correspondientes a la cinta amurallada, y se está preparando la ejecución de una tercera, referida a la Torre del Homenaje.

Precisamente en previsión de la tercera fase de intervención, se ha llevado a cabo el presente estudio sobre la Torre, con el objetivo de estudiar y documentar el estado actual del edificio para realizar una base de conocimiento para la redacción del proyecto de restauración.

\subsection{La Torre del Homenaje del Castillo de Cofrentes}

$\mathrm{El}$ asentamiento de Cofrentes se ubica en el valle de Ayora en una de las colinas a occidente respecto al río. El Castillo ocupa, con sus murallas, la cima de una gran roca volcánica, que el río Cabriel ha ido tallando con el tiempo. Está situado en posición estratégica, abierto hacia el valle con la Torre para el control del mismo, y completamente protegido hacia norte por un profundo barranco creado por el mismo río. Si para el castillo se estima posible un origen

\footnotetext{
${ }^{6} \mathrm{El}$ análisis estratigráfico constructivo de la Torre del Homenaje del Castillo de Cofrentes ha sido desarrollado, en el año 1998, por los autores en el ámbito del encargo "Realización estudio estratigráfico para la restauración de la Torre del Homenaje del Castillo, en Cofrentes (V)», asignado al arquitecto Francisco Cervera Arias por la Consellería de Cultura y Ciencia de la Generalitat Valenciana. Camilla Mileto ha tenido, además, la posibilidad de colaborar a la redacción del proyecto de restauración, en el año 1999, en colaboración con el mismo arquitecto Cervera, encargado del proyecto.
}

entre finales del siglo X y principios del siglo XI, —aunque luego haya sufrido una serie de intervenciones hasta el siglo XIX-, para la torre, sin embargo, no existen documentos o restos que permitan fijar fielmente una fecha anterior al siglo XVI para su construcción.

La Torre del Homenaje, que se ubica en posición central respecto a la cinta interior de las murallas, muestra desde abajo toda su imponencia. La fábrica está construida en mampostería con esquinas de sillería hasta los tres cuartos de la altura de la torre, para seguir con una construcción de tapia en la parte superior. Desde el interior del castillo, la torre aparece mucho más pequeña que desde el exterior, ya que parte de su paramento exterior está adosado a la montaña, con el objetivo de doblar casi la altura total de la torre, para aparentar mayor fuerza y potencia.

En el interior, la torre consta de tres pisos, de los cuales, la planta baja consiste en una estancia abovedada, mientras que los otros dos estaban divididos por un forjado, actualmente en gran parte caído.

\subsection{La metodología adoptada}

La Torre se presenta como un único cuerpo de fábrica, compacto, aunque haya sufrido, en el tiempo, una serie de intervenciones, sobre todo ligadas, por un lado, a la sobreelevación de la parte superior, y por otro lado a modificaciones de los acabados y de los huecos.

La Torre presenta, por esta razón, una notable estratificación de intervenciones de modificación, tanto en lo que respecta a los elementos constructivos, como en lo que atañe a las superficies de acabado. El estudio se dirige, por ello, a los paramentos del edificio, tanto internos como externos y a la identificación de las relaciones que existen entre las diversas partes heterogéneas reconocidas.

Se han elaborado una serie de alzados generales (para los cuatro alzados exteriores) y una serie de alzados de detalle, tanto como mayor nivel de detalle de los mismos alzados generales, como para el estudio de los alzados interiores. En cada uno se han identificado las zonas homogéneas como unidades estratigráficas constructivas.

\subsection{Descripción de la hipótesis de los periodos constructivos de la Torre del Homenaje}

Se han identificado sustancialmente seis periodos constructivos para los que se propone una hipótesis de arco temporal que no pretende o intenta brindar una interpretación histórica. Se trata de hecho de una cronología relativa, o sea, una periodización correspondiente solamente a las unidades estratigráficas presentes en el edificio y que no ofrece una datación precisa, dada la falta de información histórica comprobada. 


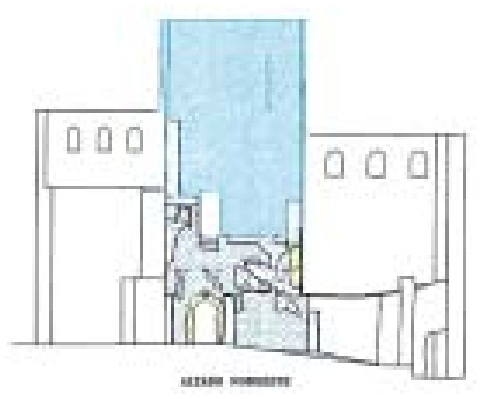

LETENaA

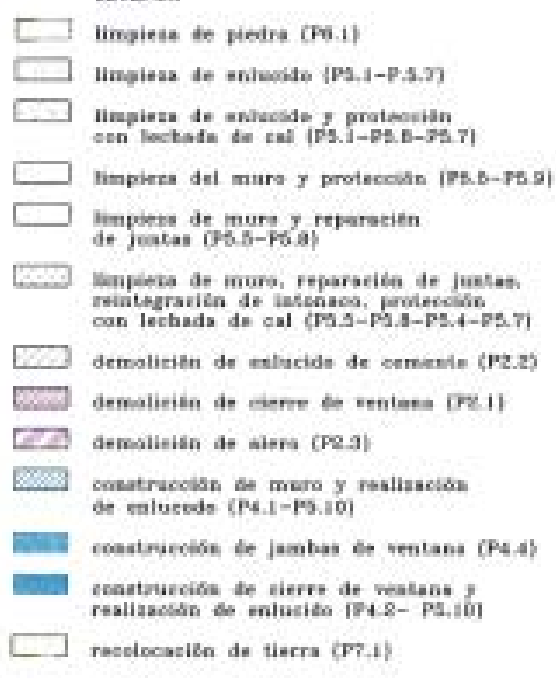

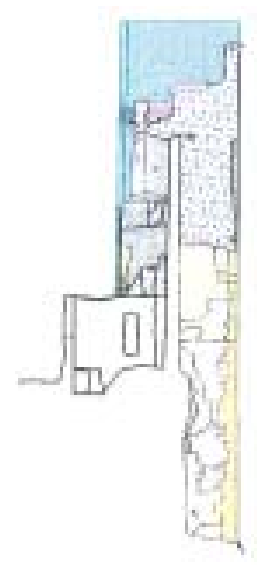

une nom
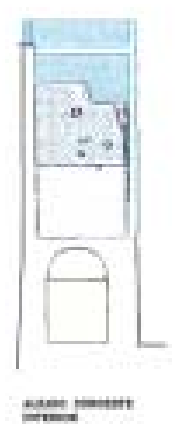
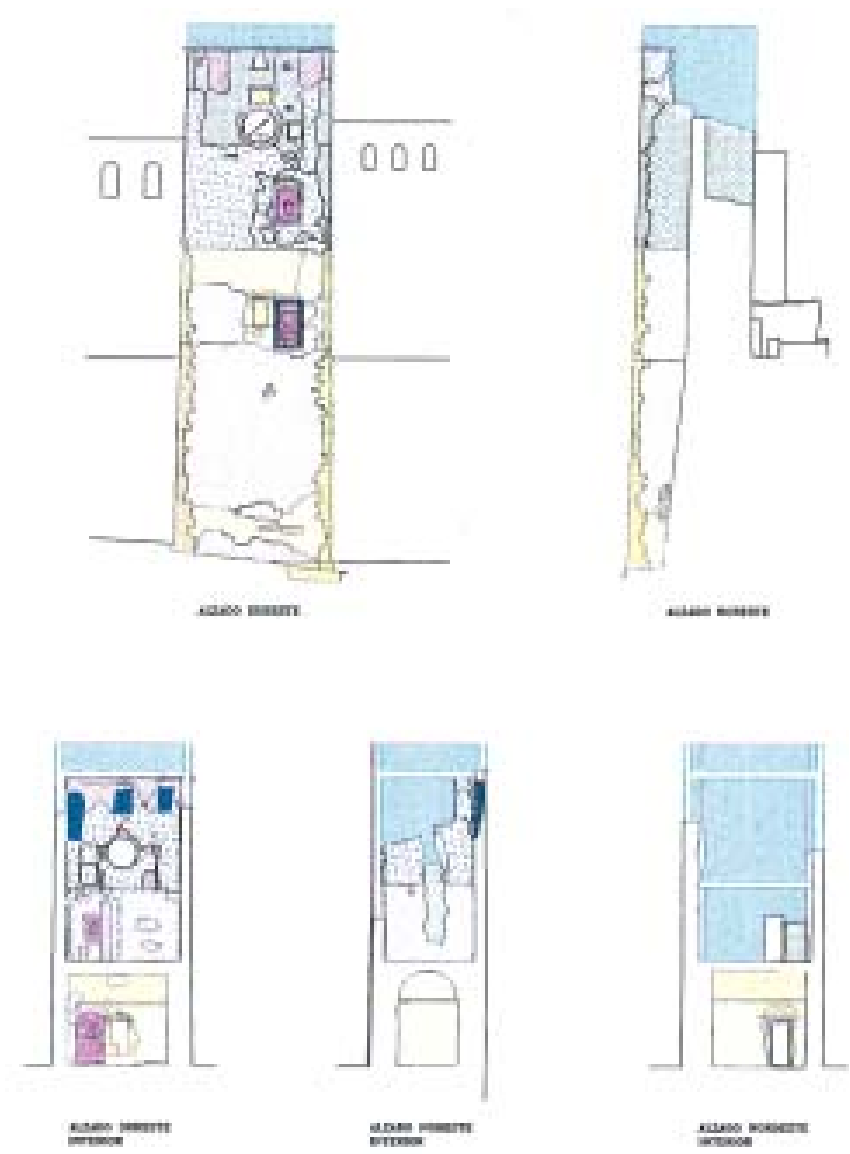

Fig. 3. Plano del proyecto de superficies de la Torre del Homenaje del castillo de Cofrentes (Valencia)

\section{Periodo 1}

Se trata del periodo en el que se ha construido la primera parte de la torre compuesta de esquinas de sillares de piedra caliza de color claro, y muros de mampostería de piedra caliza y piedra volcánica, tomados con mortero de cal. En los alzados se puede observar en las esquinas y en el paramento hasta más de la mitad de la altura de la fábrica. A este primer periodo se corresponde casi en su totalidad, salvo algunas modificaciones, la estancia abovedada de la planta baja.

\section{Periodo 2}

Se trata de la redefinición de los paramentos externos del edificio ya existente, y se caracteriza sobre todo por la aportación de un revoco de cal. Aunque se ha evidenciado como un II periodo, es posible de todas maneras que este revoco fuera el tratamiento superficial del paramento ya en el primer periodo.

\section{Periodo 3}

También en este caso se trata de un periodo principalmente de redefinición de los paramentos externos y de pequenas modificaciones que podrían formar parte del siguiente periodo IV. Dada la ausencia de datos incontrovertibles, se ha pensado en separar este periodo del siguiente, y de senalar la duda de la división con una línea a trazos. Se caracteriza sobre todo por la aportación sobre los muros externos de un estrato posterior de enlucido. Se trata de un enlucido (de cal). Este tratamiento de acabado se puede distinguir en tres lados externos de la torre (sureste, noreste, suroeste), y sólo a mitad de la altura del cuerpo de la fábrica. Se trata de un estrato superpuesto al enlucido de la parte inferior de la torre y claramente diferente. Podría ser incluso una imitación hecha en un periodo posterior del enlucido ya existente.

\section{Periodo 4}

Se trata de una fase importante de actuaciones en la fábrica que consiste en la sobreelevación de la torre. El muro se construyó con técnica de tapia armada de ladrillo (valenciana), con paramentos constituidos por mortero de cal y ladrillo e interior formado por tongadas de arcilla comprimida. Superpuesto al muro se puede distinguir un estrato de enlucido de cal que constituía la protección superficial del muro. Al mismo periodo pertenecen varias acciones de mo- 
dificación de vanos existentes. En el interior se puede leer la sobreelevación de la torre que comporta la total redefinición de los espacios: la definición de nuevas estancias, la subdivisión interna con un forjado de madera, la construcción de una pequeña escalera de fábrica. Se debe señalar la posible adscripción a este periodo de la cornisa que define el volumen de la torre en la parte superior.

\section{Periodo 5}

Se trata de un periodo muy reciente que consistió sobre todo en pequeños remiendos hechos con enfoscado de cemento, la inserción del reloj y la clausura de algunos vanos. En el lado noroeste se leen las trazas de una pequeña escalera adscribible a este periodo, para facilitar el acceso al primer nivel y que, posteriormente, fue destruida. Siempre parte de este periodo se considera la demolición de parte de la torre ocurrida en 1992 a causa de la precariedad estructural del edificio. Esta intervención es legible hoy en día en el mortero de yeso que se ha extendido como protección en las crestas de los muros desmochados.

\section{Periodo 6}

En la parte interna del alzado suroeste se puede leer la construcción de un muro de conexión entre la torre y la muralla del recinto. Esta intervención se corresponde con la restauración de los muros laterales de la torre.

\subsection{La relación entre el análisis estratigráfico constructivo y el proyecto de restauración (Fig. 3)}

Como se ha comentado al principio, la lectura estratigráfica realizada para la Torre del Homenaje del Castillo de Cofrentes se insertaba en el marco de un estudio que tenía como objetivo principal el conocimiento del edificio para un sucesivo proyecto de restauración. La toma de datos materiales (materiales empleados, elaboración, técnicas constructivas, etc.) y de las relaciones que se establecen entre ellos, por su capacidad de registrar y evidenciar la importancia y la unicidad de las señales dejadas por las acciones individuales, asume un papel fundamental en el momento de formular un proyecto de restauración consciente, que esté en grado de respetar y conservar estos mismos datos materiales y garantizar su transmisión al futuro.

El objetivo común del análisis estratigráfico y el proyecto de restauración debe por tanto ser el estudio de la materialidad del edificio para la conservación de la misma. Conservación de la materia, transmisibilidad de las relaciones estratigráficas y legibilidad de la intervención deben garantizarse mediante una elección adecuada de la modalidad del proyecto. No se trata de congelar el edificio, sino más bien de concebir la intervención como una fase más en la estratificación. Se trata de definir los modos de contacto entre lo existente y lo nuevo de manera que se respeten los materiales, las técnicas constructivas, los significados, el espíritu de lo existente.

El proyecto de restauración para la Torre del Homenaje ha sido redactado con la mirada puesta siempre en el estudio estratigráfico. Sin embargo, el proyecto no rechaza absolutamente la inserción del nuevo (la escalera, el forjado, los cierres de las ventanas, la cubierta, etc.), sino más bien asume en cada momento una actitud de respeto de lo existente, tanto en los materiales (paramento, enlucidos, etc.) como en las estructuras. En especial modo, el proyecto de conservación de las superficies se ha redactado con el objetivo de la total conservación de las huellas del tiempo (enlucidos diferentes, morteros diferentes, fábricas y mamposterías, etc.). Se trata entonces de compaginar una consolidación de lo existente con la inserción de elementos de nueva planta que le permitan desempeñar las funciones de museo de sí misma y plataforma panorámica, asignadas al edificio. 